\title{
THE LATE PEAKING AFTERGLOW OF GRB 100418A
}

\author{
F. E. Marshall ${ }^{1}$, L. A. Antonelli ${ }^{2}$, D. N. Burrows ${ }^{3}$, S. Covino ${ }^{4,5}$, M. De Pasquale ${ }^{6}$, P. A. Evans ${ }^{7}$, D. Fugazza ${ }^{4}$, \\ S. T. Holland ${ }^{1,8,9}$, E. W. Liang ${ }^{10,11}$, P. T. O’Brien ${ }^{7}$, S. R. OAtes ${ }^{6}$, J. P. Osborne ${ }^{7}$, C. Pagani ${ }^{7}$, T. Sakamoto ${ }^{1,8,12}$, \\ M. H. SIEGEL ${ }^{3}$, X. F. Wu ${ }^{10,13}$, AND B. ZhANG ${ }^{10}$ \\ ${ }^{1}$ Astrophysics Science Division, NASA Goddard Space Flight Center, Greenbelt, 20771 MD, USA; frank.marshall@ nasa.gov \\ ${ }^{2}$ INAF-Astronomical Observatory of Rome, via Frascati 33, 00040 Monteporzio (Rome), Italy \\ ${ }^{3}$ Department of Astronomy and Astrophysics, Pennsylvania State University, 525 Davey Laboratory, University Park, PA 16802, USA \\ ${ }^{4}$ INAF-Osservatorio Astronomico di Brera, via Emilio Bianchi 46, 23807, Merate (LC), Italy \\ ${ }^{5}$ INAF/TNG Fundación Galileo Galilei - Rambla José Ana Fernández Pérez, 7, 38712, Breña Baja, TF, Spain \\ ${ }^{6}$ Mullard Space Science Laboratory (UCL), Holmbury Road, Holmbury St Mary, Dorking RH5 6NT, UK \\ ${ }^{7}$ Department of Physics and Astronomy, University of Leicester, University Road, Leicester LE1 7RH, UK \\ ${ }^{8}$ Center for Research and Exploration in Space Science and Technology, NASA/GSFC, Greenbelt, 20771 MD, USA \\ ${ }^{9}$ Universities Space Research Association, 10211 Wincopin Circle, Columbia, MD 21044, USA \\ ${ }^{10}$ Department of Physics and Astronomy, University of Nevada, Las Vegas, NV 89154-4002, USA \\ ${ }^{11}$ Department of Physics, Guangxi University, Guangxi 530004, China \\ 12 Joint Center for Astrophysics, University of Maryland, Baltimore County, 1000 Hilltop Circle, Baltimore, MD 21250, USA \\ ${ }^{13}$ Purple Mountain Observatory, Chinese Academy of Sciences, Nanjing 210008, China \\ Received 2010 September 16; accepted 2010 November 25; published 2011 January 12
}

\begin{abstract}
GRB 100418A is a long gamma-ray burst (GRB) at redshift $z=0.6235$ discovered with the Swift Gamma-ray Burst Explorer with unusual optical and X-ray light curves. After an initial short-lived, rapid decline in X-rays, the optical and X-ray light curves observed with Swift are approximately flat or rising slightly out to at least $\sim 7 \times 10^{3}$ $\mathrm{s}$ after the trigger, peak at $\sim 5 \times 10^{4} \mathrm{~s}$, and then follow an approximately power-law decay. Such a long optical plateau and late peaking is rarely seen in GRB afterglows. Observations with Rapid Eye Mount during a gap in the Swift coverage indicate a bright optical flare at $\sim 2.5 \times 10^{4} \mathrm{~s}$. The long plateau phase of the afterglow is interpreted using either a model with continuous injection of energy into the forward shock of the burst or a model in which the jet of the burst is viewed off-axis. In both models the isotropic kinetic energy in the late afterglow after the plateau phase is $\geqslant 10^{2}$ times the $10^{51}$ erg of the prompt isotropic gamma-ray energy release. The energy injection model is favored because the off-axis jet model would require the intrinsic $T_{90}$ for the GRB jet viewed on-axis to be very short, $\sim 10 \mathrm{~ms}$, and the intrinsic isotropic gamma-ray energy release and the true jet energy to be much higher than the typical values of known short GRBs. The non-detection of a jet break up to $t \sim 2 \times 10^{6} \mathrm{~s}$ indicates a jet half-opening angle of at least $\sim 14^{\circ}$, and a relatively high-collimation-corrected jet energy of $E_{\text {jet }} \geqslant 10^{52} \mathrm{erg}$.
\end{abstract}

Key words: gamma-ray burst: individual (GRB 100418A)

Online-only material: color figures

\section{INTRODUCTION}

For a brief time each gamma-ray burst (GRB) becomes the most luminous object in the universe, typically releasing $10^{50}-10^{52}$ erg in $\gamma$-rays during the prompt emission phase, which can last from a few milliseconds to thousands of seconds. The prompt emission is followed by the afterglow, which is typically first detected a few tens of seconds after the start of the prompt phase and can last for several months. The energy of the burst is transported in a relativistic jet that is thought to radiate via both internal shocks that produce the prompt emission and external shocks that produce the afterglow (see reviews such as Piran 2005; Mészáros 2006).

There are two known classes of GRBs (Kouveliotou et al. 1993), which are classified according to the duration and spectrum of the prompt emission. Most of the GRBs discovered with Swift fall into the long/soft category, and this paper deals exclusively with long GRBs. Such bursts typically have durations of $\gtrsim 2 \mathrm{~s}$ in the observer's frame and are thought to be due to the collapse of rotating massive stars (Woosley 1993).

The launch and successful operation of the Swift GRB Explorer (Gehrels et al. 2004) made it possible for the first time to closely monitor the light curves of dozens of GRBs from the beginning of the prompt emission through the early afterglow and continuing for days and sometimes weeks later. Swift includes a wide-field instrument, the Burst Alert Telescope (BAT; Barthelmy et al. 2005), which covers the 15-350 keV energy range, and two narrow-field instruments, the $\mathrm{X}$-Ray Telescope (XRT; Burrows et al. 2005), which covers the $0.2-10 \mathrm{keV}$ energy range, and the Ultraviolet/Optical Telescope (UVOT; Roming et al. 2005), which covers the 170-650 $\mathrm{nm}$ wavelength range. The BAT is designed to detect GRBs within its 1.4 sr (halfcoded) field of view, which triggers a rapid, autonomous slew of the observatory to point the XRT and UVOT at the burst.

An examination of the first 27 long GRBs well observed with the XRT revealed (Nousek et al. 2006; Zhang et al. 2006) a canonical shape for the X-ray light curve consisting of three power-law segments: a rapidly falling initial segment followed by a very shallow decay and then by a steeper decay. X-ray flares were commonly superimposed on this canonical light curve. The transition from the first to second segment typically occurred $\lesssim 500 \mathrm{~s}$, and the transition from the second to the third segment typically occurred between $\sim 10^{3}$ and $\sim 10^{4} \mathrm{~s}$.

The X-ray light curves are generally understood as follows. The early rapid decay component is the tail of the prompt emission likely due to the "curvature effect" of the high-latitude emission. The following segment with its very shallow decay is 
likely due to the continuing injection of energy into the forward shock perhaps by continuing activity of the central engine or by the late arrival of jet material with lower Lorentz factors. The transition to a steeper decay slope is thought to be due to the cessation of this continuing energy injection (Zhang et al. 2006; Nousek et al. 2006).

The optical light curve of GRB 100418A is unusual and features a long initial plateau that lasts from the first UVOT observation $87 \mathrm{~s}$ after the BAT trigger until at least $\sim 7 \times 10^{3} \mathrm{~s}$ after the trigger, followed by a poorly sampled increase in flux, and then a decay that passes through the initial plateau level at $\sim 1 \times 10^{5}$ s after the trigger. Recent studies of the broad sample of optical afterglows found that for the vast majority, unlike for GRB 100418A, the optical flux peaks within $\sim 550$ s of the GRB trigger. The study by Panaitescu \& Vestrand (2008) of the optical light curves of 30 GRBs with known redshifts found a broad range of characteristics. There were four categories for the light curves: fast risers (six GRBs), slow risers (five), decays (12), and plateaus (six). One GRB could be either a fast or slow riser. For all but two of the GRBs, the peak flux occurred at $\leqslant 550$ s. The peak for GRB 060614 (Della Valle et al. 2006; Mangano et al. 2007) was at $21 \mathrm{ks}$, and the peak for GRB 070110 (Troja et al. 2007) was at $5 \mathrm{ks}$. Included in the plateau category was GRB 060729, whose X-ray afterglow was monitored for 125 days with Swift (Grupe et al. 2007). The plateau phase lasted until $\sim 50 \mathrm{ks}(\sim 60 \mathrm{ks})$ after the trigger in the optical (X-ray) band. Panaitescu \& Vestrand (2008) concluded that the range in characteristics in their sample could be explained by angular structure in the relativistic outflow and the likely range jet orientations to the observer's line of sight. GRB 070311 was not included in the sample of Panaitescu \& Vestrand (2008) because its redshift is not known, but it shows a re-brightening in X-ray and optical bands between $3 \times 10^{4}$ and $2 \times 10^{5} \mathrm{~s}$ after the burst (Guidorzi et al. 2007), which was interpreted as being due to a refreshed external shock.

A later statistical study (Oates et al. 2009) of 26 long GRBs well observed with the UVOT (including 10 overlapping with those in the Panaitescu \& Vestrand (2008) sample) found very different characteristics for the optical/ultraviolet light curves than those of the X-ray light curves. Most decayed from the start of the observations (typically within $100 \mathrm{~s}$ ), but at least six of the GRBs have an initially rising light curve. All decayed after $500 \mathrm{~s}$, typically as a power law with a mean index $\alpha$ of 0.88 and a standard deviation of 0.31 . GRB 060614 and GRB 070110 were not included in the sample because of the selection criterion that the flux in the $V$ filter be brighter than $17.8 \mathrm{mag}$. Large flares, such as those seen with the XRT, were not observed, but a rebrightening of $\sim 1$ mag starting at $2.9 \times 10^{3} \mathrm{~s}$ after the trigger was seen for GRB 060206 (see also Woźniak et al. 2006; Stanek et al. 2007). Oates et al. (2009) concluded that the rising optical light curves are most likely caused by the start of the forward shock, although there may be cases in which the rise is due to viewing the jet off-axis.

Late optical re-brightening on timescales comparable to that seen for GRB 100418A has been reported for two recent GRBs. GRB 080129 (Greiner et al. 2009) has an optical light curve increasing from $t \sim 1 \mathrm{ks}$ to at least $t \sim 6 \mathrm{ks}$ since the trigger. Shen et al. (2010) suggested that the very late re-brightening in this burst could be caused by the interaction of a late jet and a cocoon created by the main jet. The optical afterglow GRB 081028 brightened from about $10 \mathrm{ks}$ to $50 \mathrm{ks}$ after the trigger, which Margutti et al. (2010) interpreted as a narrow jet being viewed off-axis.
GRB 100418A was discovered with the Swift BAT triggered (trigger 419797; Marshall et al. 2010) on 2010 April 18 at 21:10:08 UT $\left(=T_{0}\right)$ and located the burst at the coordinates $\alpha=17^{\mathrm{h}} 05^{\mathrm{m}} 25^{\mathrm{s}} .8, \delta=+11^{\circ} 27^{\prime} 26^{\prime \prime} .8$ (equinox 2000.0 ) with an estimated uncertainty of 1.9 (90\% containment including systematic error; Ukwatta et al. 2010). Its long duration and soft spectrum (Section 2.1) clearly show it to be a long burst. The observatory immediately slewed to the burst and XRT and settled UVOT observations began $79 \mathrm{~s}$ and $87 \mathrm{~s}$, respectively, after the trigger.

The XRT found a fading, uncataloged source within the BAT's error region. The source decayed rapidly until about $T_{0}+474 \mathrm{~s}$, after which the light curve flattened to a decay index of 0.21 (Pagani et al. 2010).

The UVOT found an afterglow candidate in its initial, $150 \mathrm{~s}$ finding chart exposure in the white (160-800 nm) filter, which started at $T_{0}+87 \mathrm{~s}$. No evidence for fading was seen in the first two orbits of data, which ended at $T_{0}+7.3 \mathrm{ks}$. The initial white magnitude was $21.6 \pm 0.3$ (Siegel \& Marshall 2010). Preliminary analysis detected the afterglow in all the UVOT filters including a marginal $(2.5 \sigma)$ detection with the $v$ filter. The detection in the $u v w 2$ filter indicated a low redshift burst with low dust extinction.

The optical afterglow was observed with numerous groundbased observatories including the robotic Rapid Eye Mount (REM) 60-cm telescope located in La Silla, Chile (Covino et al. 2010). A redshift of 0.6235 was found with the Very Large Telescope (Antonelli et al. 2010), and later confirmed with the Gemini North telescope (Cucchiara et al. 2010). Malesani (2010) reported the detection of the host galaxy in the Sloan Digital Sky Survey data.

In this paper, we present and discuss the optical, UV, and X-ray emission properties of this unusual GRB as observed by Swift and the REM. Details of the BAT, XRT, UVOT, and REM observations are given in Section 2. The temporal and spectral analysis results are reported also in Section 2, and theoretical interpretations are given in Section 3. Finally, Section 4 provides a summary.

Uncertainties quoted in this paper are given at the $90 \%$ confidence level for one interesting parameter unless otherwise noted. We adopt the notation $F(v, t)=t^{-\alpha} v^{-\beta}$ for the monochromatic flux as a function of time. The photon index $\Gamma$ is then $\beta+1$. A standard cosmology model with $H_{0}=70 \mathrm{~km} \mathrm{~s}^{-1}, \Omega_{M}=0.3$, and $\Omega_{\Lambda}=0.7$ is used.

\section{OBSERVATIONS AND DATA REDUCTION}

The XRT and UVOT observed the afterglow regularly for almost 29 days using sequence numbers 00419797000 through 00419797032. There was one final observation five days later. The total UVOT exposure was $219 \mathrm{ks}$ (including $189 \mathrm{ks}$ with the white filter), and the total XRT exposure was $232 \mathrm{ks}$.

\subsection{Swift BAT Observations}

Figure 1 shows the BAT light curves. The prompt emission consists of two main overlapping peaks. The lack of obvious emission above $100 \mathrm{keV}$ indicates the soft nature of the burst. The $T_{90}$ duration is $8 \pm 2 \mathrm{~s}$ in the $15-150 \mathrm{keV}$ band. The timeintegrated spectrum during the prompt phase is best fit by a single power law with the photon index $\Gamma=2.16 \pm 0.25$. There is no significant improvement in $\chi^{2}$ for the fit for a cutoff powerlaw model over a simple power-law model. The $15-150 \mathrm{keV}$ fluence is $(3.4 \pm 0.5) \times 10^{-7} \mathrm{erg} \mathrm{cm}^{-2}$. The ratio of the fluence 


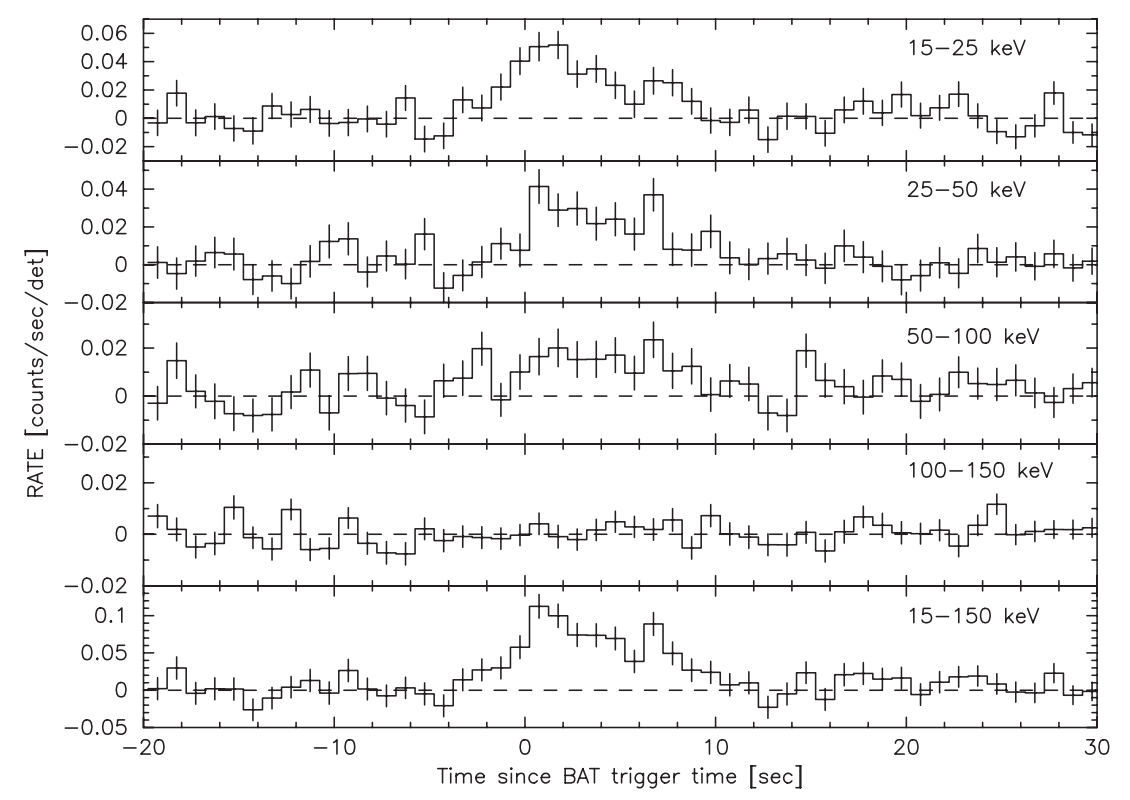

Figure 1. BAT light curves in five energy bands.

in the $25-50 \mathrm{keV}$ band to the fluence in the $50-100 \mathrm{keV}$ band is $1.12 \pm 0.29$, which indicates that GRB $100418 \mathrm{~A}$ is probably a member of the X-ray rich class of GRBs (Sakamoto et al. 2008). The $1 \mathrm{~s}$ peak flux is $(5.1 \pm 1.5) \times 10^{-8} \mathrm{erg} \mathrm{cm}^{-2} \mathrm{~s}^{-1}$ in the $15-150 \mathrm{keV}$ band.

Although $E_{\text {peak }}$ cannot be measured directly from the observed BAT spectrum, $E_{\text {peak }}$ is very likely to be located at the lower energy range of BAT since the BAT photon index of 2.16 is very close to the typical high-energy photon index of the Band function (Kaneko et al. 2006). Using the $E_{\text {peak }}-\Gamma$ relation (Sakamoto et al. 2009) and the BAT photon index of 2.16, we find $E_{\text {peak }}$ is $29_{-27}^{+2}(1 \sigma) \mathrm{keV}$.

To estimate the isotropic gamma-ray energy release $E_{\gamma}$,iso, we fit the BAT-time-integrated spectrum with the Band function (Band et al. 1993) by fixing the low-energy photon index to 1.0 , and then calculated $E_{\gamma \text {,iso }}$ in the $1 \mathrm{keV}-10 \mathrm{MeV}$ band (cosmological rest frame). The best estimate of $E_{\gamma \text {,iso }}$ is $9.9_{-3.4}^{+6.3} \times 10^{50} \mathrm{erg}$. We applied the same approach to estimate the $1 \mathrm{~s}$ peak luminosity $L_{\gamma}$,iso using the BAT $1 \mathrm{~s}$ peak spectrum. Our estimated $L_{\gamma \text {,iso }}$ is $2.1_{-0.6}^{+1.1} \times 10^{50} \mathrm{erg} \mathrm{s}^{-1}$ (in the $1 \mathrm{keV}-10$ $\mathrm{MeV}$ band in the rest frame).

\subsection{Swift XRT Observations}

The XRT started observations of GRB 100418A $71 \mathrm{~s}$ after the BAT trigger, collecting data in Windowed Timing (WT) settling mode while the spacecraft was slewing to the burst location. Swift settled on the burst $79 \mathrm{~s}$ after the trigger and the XRT autonomously localized the afterglow on-board taking in the image of the field. The astrometrically corrected X-ray position (Goad et al. 2007; Evans et al. 2009) of R.A., decl. = 256.36313, +11.46140 , equivalent to $\alpha=17^{\mathrm{h}} 05^{\mathrm{m}} 27^{\mathrm{s}} .15, \delta=+11^{\circ} 27^{\prime} 41^{\prime \prime} .1$ (equinox 2000.0) with an estimated uncertainty of $1^{\prime \prime} 9$ (radius, $90 \%$ confidence including systematic error), updated from the position reported by Osborne et al. (2010), was derived from the full, on-ground data set, using the XRT-UVOT alignment and matching UVOT field sources to the USNO-B1 catalog. Settled WT mode data were collected from $T_{0}+86 \mathrm{~s}$ to $T_{0}+174 \mathrm{~s}$; subsequently, the XRT switched into photon counting (PC) mode for the rest of the observations, from $T_{0}+182 \mathrm{~s}$ to $T_{0}+3 \mathrm{Ms}$, for a total observing time of $231.8 \mathrm{ks}$.
The XRT data were reduced with the standard software (XRTPIPELINE v0.12.4) applying the default filtering and screening criteria (HEASOFT 6.8, Swift tools 3.5), using the latest CALDB 3.6 files released in 2010 June.

The X-ray light curve in the $0.3-10 \mathrm{keV}$ energy band was extracted using the methods described in Evans et al. (2007, 2009). The overall light curve can be modeled by a doublebroken power law, as shown in Figure 2. The early steep phase is fitted with a decay index $\alpha=4.18_{-0.14}^{+0.16}$. The end of the steep decay at approximately $T_{0}+600 \mathrm{~s}$ is followed by a shallow phase that bears resemblance to the afterglow brightening observed by the UVOT. If one assumes that the observed count rate behavior is not due to superimposed X-ray flares, this afterglow phase can be fitted with a power law of index $\alpha=-0.23_{-0.14}^{+0.13}$ out to approximately $T_{0}+66 \mathrm{ks}$. The late afterglow decays with an index of $\alpha=1.37_{-0.11}^{+0.13}$. The double-broken power-law fit is statistically marginally acceptable, with a $\chi^{2}$ of 100.69 for 66 degrees of freedom $\left(\chi_{\text {red }}^{2}\right.$ of 1.52), an indication of the complexity of the X-ray emission likely due to the presence of mini-flares. A broken power-law fit of the light curve, with the exclusion of the early steep decay is statistically more acceptable $\left(\chi^{2}\right.$ of 38.82 for 25 degrees of freedom) and gives fit parameters consistent with the reported values of the double-broken power law.

We have extracted time-resolved spectra of the X-ray afterglow in the energy range $0.3-10 \mathrm{keV}$, performing the analysis using XSPEC v12.5.1n. The spectrum from the WT mode data, selecting events with grades $0-2$ and binned with 20 counts per bin was fitted with an absorbed power law (using the X-ray absorption models PHABS and ZPHABS), fixing the Galactic absorption to the value of $4.8 \times 10^{20} \mathrm{~cm}^{-2}$ (Kalberla et al. 2005). The best fit yields a steep photon index of $4.33_{-0.25}^{+0.28}$ and an intrinsic $N_{\mathrm{H}}$ of $3.3 \pm 0.6 \times 10^{21} \mathrm{~cm}^{-2}$ at a redshift of 0.6235 , in excess of the Galactic value, with a $\chi^{2}$ of 47.5 (42 degrees of freedom). The PC spectrum during the plateau phase, binned to one count per bin and using Cash statistics (Cash 1979) can be fitted with an absorbed power law with a photon spectral index of $2.09_{-0.34}^{+0.25}$ and an intrinsic absorption of $2.0_{-1.8}^{+1.6} \times 10^{21} \mathrm{~cm}^{-2}$.

To investigate the possibility of spectral evolution in the WT mode observations, we extracted an early WT spectrum from 


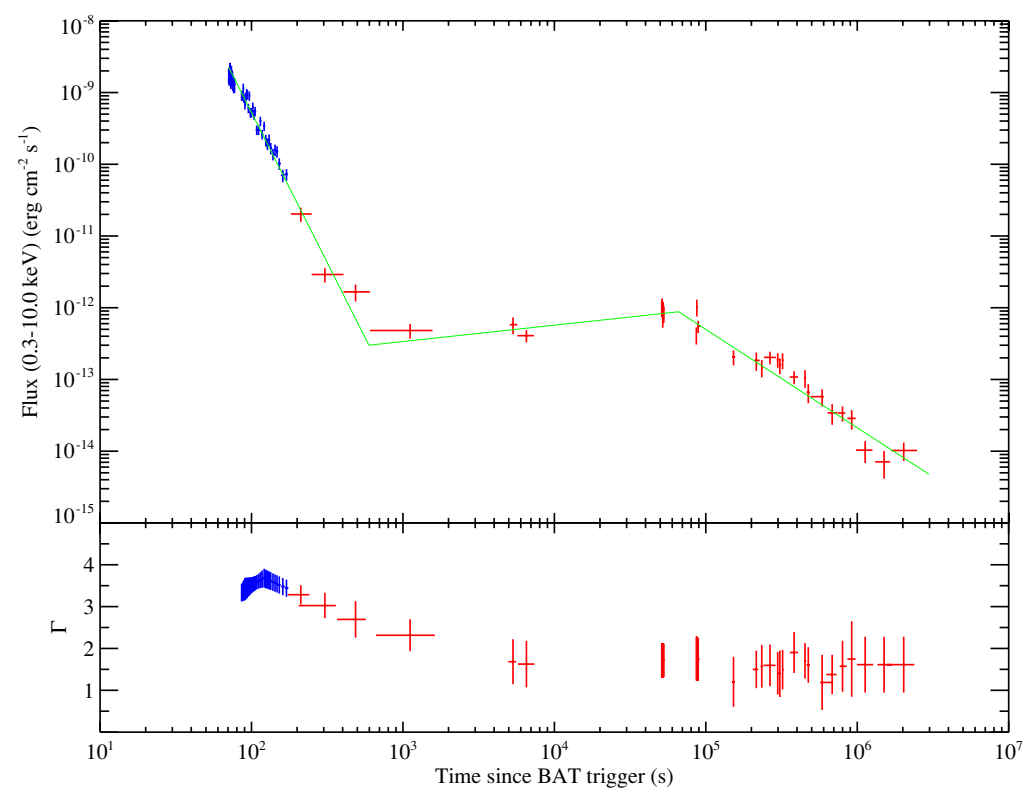

Figure 2. XRT light curve of GRB 100418A in the energy band 0.3-10 keV (top panel) and the estimated photon index $(\Gamma)$ interpolated from the observed hardness ratio (lower panel). The time bars correspond to the time bin sizes. Data from times less than $174 \mathrm{~s}$ from the trigger, taken in WT mode, are shown in blue; later data, taken in PC mode, are shown in red. The best-fit broken power-law model to the light curve is shown.

(A color version of this figure is available in the online journal.)

Table 1

X-ray Afterglow Spectral Parameters

\begin{tabular}{|c|c|c|c|c|c|c|c|c|}
\hline Phase & Time from Trigger & $\begin{array}{c}N_{\mathrm{H}}(\text { intrinsic }) \\
\text { PL }\end{array}$ & $\Gamma$ & $\chi^{2}$ & $\begin{array}{c}N_{\mathrm{H}}(\text { intrinsic }) \\
\mathrm{PL}+\mathrm{BB}\end{array}$ & $\Gamma$ & $\mathrm{kT}(\mathrm{keV})$ & $\chi^{2}$ \\
\hline WT & $84 \mathrm{~s}<T<174 \mathrm{~s}$ & $3.3 \pm 0.6 \times 10^{21} \mathrm{~cm}^{-2}$ & $4.33_{-0.25}^{+0.28}$ & 47.5 (42 dof) & $1.2_{-1.1}^{+1.5} \times 10^{21} \mathrm{~cm}^{-2}$ & $2.46_{-1.12}^{+1.05}$ & $0.12 \pm 0.02$ & 32.5 (40 dof) \\
\hline $\mathrm{PC}$ & $600 \mathrm{~s}<T<66 \mathrm{ks}$ & $2.0_{-1.8}^{+1.6} \times 10^{21} \mathrm{~cm}^{-2}$ & $2.09_{-0.34}^{+0.25}$ & $81.43(108)^{\mathrm{a}}$ & $\cdots$ & $\cdots$ & $\cdots$ & $\cdots$ \\
\hline
\end{tabular}

Notes. Spectral and timing parameters of the X-ray afterglow evolving phases. The spectra were extracted in the energy range $0.3-10 \mathrm{keV}$ with an absorbed power law and with an absorbed power law plus blackbody model, fixing the Galactic absorption to the value of $4.8 \times 10^{20} \mathrm{~cm}^{-2}$.

${ }^{\text {a }}$ Value obtained with C-statistics fit.

$T_{0}+84 \mathrm{~s}$ to $T_{0}+103 \mathrm{~s}$ and a late spectrum from $T_{0}+103 \mathrm{~s}$ to $T_{0}+174 \mathrm{~s}$. The absorbed power-law fits to the spectra indicated a spectral softening during the WT steep decay, yielding photon indices of $4.03 \pm 0.15$ and $4.63 \pm 0.16$ for the early and late spectra, respectively, with the intrinsic absorption tied to the best-fit value of the combined data. The evolution of the photon index $\Gamma$, interpolated from the hardness ratio measurements as described in Evans et al. (2010), is shown in the bottom panel of Figure 2. A likely explanation for the measured early softening is the passage of $E_{\text {peak }}$ through the XRT energy band, as discussed by Butler \& Kocevski (2007) and suggested for this burst by the low $E_{\text {peak }}$ value derived from the BAT data. Alternatively, the difference in photon indices between the WT and the PC fits and the soft WT spectrum could be due to the presence of a blackbody component during the WT observations. The best fit to the entire WT mode data set with an absorbed power law plus blackbody model yielded a photon index of $2.46_{-1.12}^{+1.05}$, a temperature of $0.12 \pm 0.02 \mathrm{keV}$, and an intrinsic $N_{\mathrm{H}}$ of $1.2_{-1.1}^{+1.5} \times 10^{21} \mathrm{~cm}^{-2}$, with a $\chi^{2}$ of 32.5 (40 dof) and a ratio of the flux of the blackbody component over the total flux in the $0.3-10 \mathrm{keV}$ energy band equal to 0.625 . The improved $\chi^{2}$ is suggestive of a curved spectrum, but the $\chi_{\text {red }}^{2}$ of 0.81 is indicative of an issue of overfitting of the data with a spectral model with too many free parameters. We report the best-fit results with the power law and power law plus blackbody models in Table 1.
Finally, we extracted three spectra from the PC data at specific times of the afterglow emission, during the early plateau phase around $T_{0}+6 \mathrm{ks}$, at the peak of the UVOT light curve and during the late decay to produce broadband spectral energy distribution (SED) in combination with UVOT observations. The results of the combined fits are presented in Section 2.4.

\subsection{Swift UVOT Optical/UV Observations}

The HEASOFT ${ }^{14}$ (v6.9) and Swift software (v3.6, Build 25) tools and the latest calibration products were used to analyze the UVOT data. The photometry was performed following the methods described by Poole et al. (2008).

The best Swift position for the afterglow was determined using the $1746 \mathrm{~s}$ exposure that started at $T_{0}+51217 \mathrm{~s}$, when the afterglow was near its peak intensity. The tool UVOTDETECT determined a position of $\alpha=17^{\mathrm{h}} 05^{\mathrm{m}} 27^{\mathrm{s}} .11, \delta=+11^{\circ} 27^{\prime} 42^{\prime \prime} .5$ (equinox 2000.0) with an estimated uncertainty of $0.43(90 \%$ containment including systematic error). The uncertainty is dominated by systematics (Breeveld et al. 2010). This position was used for all UVOT analysis reported in this paper. It supercedes the preliminary UVOT position reported by Siegel \& Marshall (2010).

UVOT photometry was performed using a $3^{\prime \prime}$ radius aperture centered on the above position. A nearby source-free region

\footnotetext{
14 http://heasarc.gsfc.nasa.gov/docs/software/lheasoft/
} 


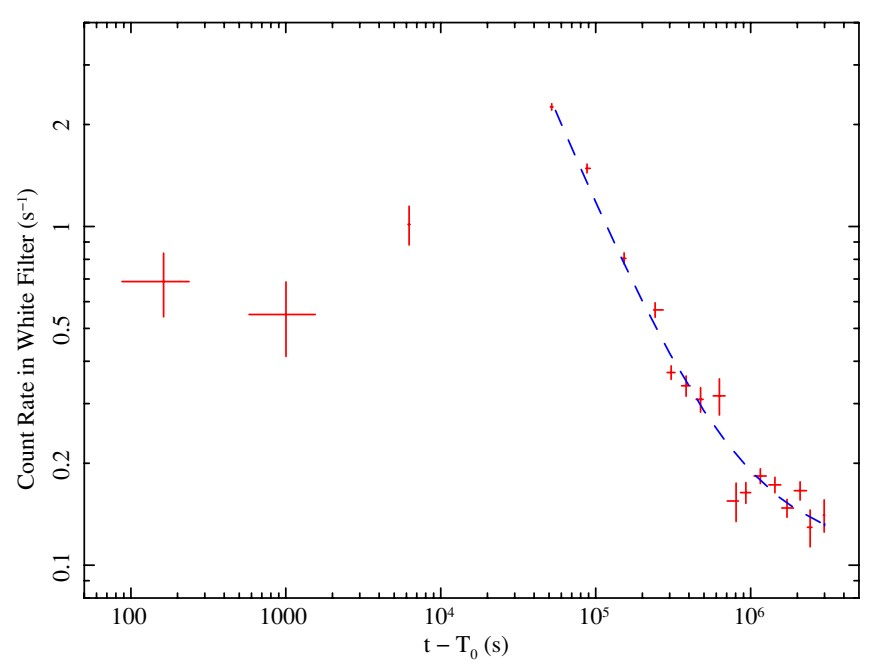

Figure 3. Swift UVOT count rate in the white filter as a function of time since the BAT trigger. When necessary for a significant detection, data from multiple exposures are included in a single data point. The dashed line is the best-fit curve to the data after $T_{0}+50 \mathrm{ks}$ to a model with a constant plus a power-law decay.

(A color version of this figure is available in the online journal.)

Table 2

UVOT Light Curve for White Filter

\begin{tabular}{|c|c|c|}
\hline $\begin{array}{l}t-T_{0} \\
\text { (1) }\end{array}$ & $\begin{array}{c}\text { Count Rate } \\
\text { (2) }\end{array}$ & $\overline{\text { Magnitude }}$ \\
\hline $0.162 \pm 0.075$ & $0.69 \pm 0.15$ & $20.70 \pm 0.21$ \\
\hline $1.00_{-0.42}^{+0.55}$ & $0.55 \pm 0.14$ & $20.94 \pm 0.24$ \\
\hline $6.24 \pm 0.10$ & $1.01 \pm 0.13$ & $20.27 \pm 0.13$ \\
\hline $51.99 \pm 1.00$ & $2.25 \pm 0.049$ & $19.41 \pm 0.02$ \\
\hline $87.93_{-1.73}^{+4.26}$ & $1.48 \pm 0.04$ & $19.86 \pm 0.03$ \\
\hline $152.41_{-3.12}^{+4.06}$ & $0.81 \pm 0.03$ & $20.52 \pm 0.04$ \\
\hline $241.67_{-5.32}^{+30.83}$ & $0.57 \pm 0.03$ & $20.91 \pm 0.05$ \\
\hline $306.72_{-17.58}^{+17.69}$ & $0.37 \pm 0.02$ & $21.37 \pm 0.05$ \\
\hline $382.86_{-24.71}^{+23.39}$ & $0.34 \pm 0.02$ & $21.46 \pm 0.07$ \\
\hline $475.13_{-30.27}^{+17.58}$ & $0.31 \pm 0.03$ & $21.57 \pm 0.09$ \\
\hline $628.62_{-55.30}^{+54.69}$ & $0.32 \pm 0.04$ & $21.54 \pm 0.12$ \\
\hline $805.65_{-99.14}^{+28.16}$ & $0.15 \pm 0.02$ & $22.32 \pm 0.13$ \\
\hline $931.36_{-69.87}^{+69.37}$ & $0.16 \pm 0.01$ & $22.25 \pm 0.07$ \\
\hline $1154.16_{-102.65}^{+105.70}$ & $0.18 \pm 0.01$ & $22.13 \pm 0.05$ \\
\hline $1433.53_{-121.97}^{+129.04}$ & $0.17 \pm 0.01$ & $22.20 \pm 0.06$ \\
\hline $1715.75_{-126.58}^{+176.28}$ & $0.15 \pm 0.01$ & $22.37 \pm 0.06$ \\
\hline $2084.83_{-171.89}^{+205.47}$ & $0.17 \pm 0.01$ & $22.24 \pm 0.07$ \\
\hline $2419.45_{-94.27}^{+66.78}$ & $0.13 \pm 0.02$ & $22.51 \pm 0.13$ \\
\hline $2983.13_{-35.15}^{+18.35}$ & $0.14 \pm 0.02$ & $22.42 \pm 0.11$ \\
\hline
\end{tabular}

Notes. (1) Mean time since the BAT trigger in units of ks; (2) Rate in counts $\mathrm{s}^{-1}$;

centered at $\alpha=17^{\mathrm{h}} 05^{\mathrm{m}} 25^{\mathrm{s}} .63, \delta=+11^{\circ} 27^{\prime} 05^{\prime \prime}$. 9 with a $9^{\prime \prime}$ radius was used for background. The standard aperture correction was used to correct for source photons falling outside the aperture. The tool UVOTPRODUCT, which rebins data to produce statistically significant data points when needed, was used to produce the light curve for the white filter reported in Table 2 and plotted in Figure 3. The count rate is approximately constant out to $10 \mathrm{ks}$, but observations after $T_{0}+50 \mathrm{ks}$ found a brighter, decaying source. For an assumed power-law decay model for the data before $T_{0}+10 \mathrm{ks}$, the best-fit decay index is $-0.15_{-0.27}^{+0.21}$. A two-component model with a constant plus a power-law decay

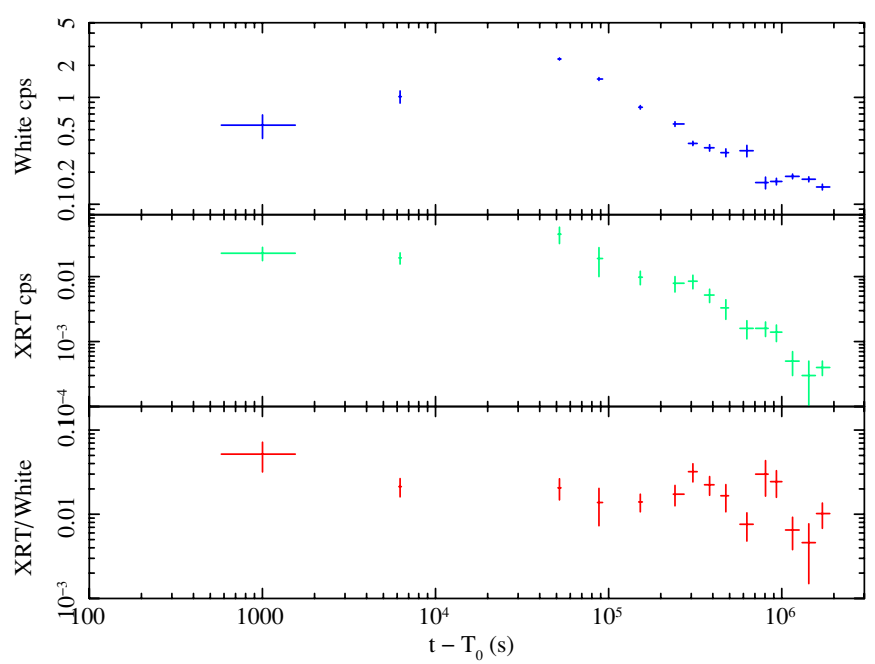

Figure 4. Swift UVOT count rate in the white filter (top panel), the XRT count rate in the $0.3-10 \mathrm{keV}$ band (middle panel), and their ratio (lower panel) as a function of time since the BAT trigger.

(A color version of this figure is available in the online journal.)

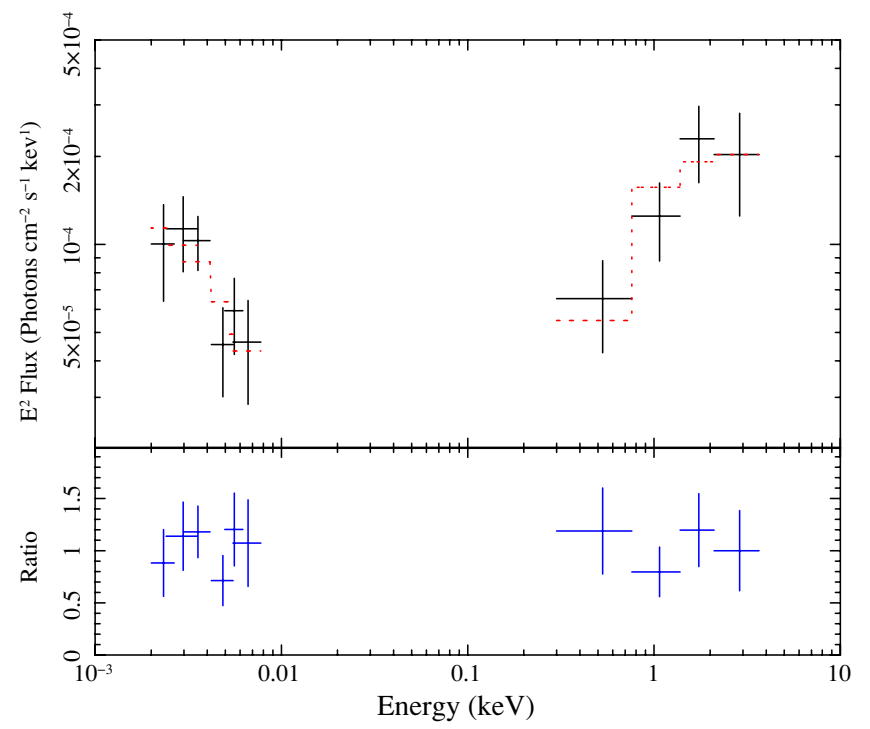

Figure 5. Best-fit combined Swift XRT and UVOT spectrum accumulated between $5.1 \mathrm{ks}$ and $7.3 \mathrm{ks}$ after the BAT trigger. The top panel shows the unfolded spectrum multiplied by $\mathrm{E}^{2}$. The dashed line shows the best-fit model. The lower panel shows the ratio of the data to the best-fit model.

(A color version of this figure is available in the online journal.)

was fit to the later data as shown in Figure 3. The best-fit decay index was $1.12 \pm 0.10$, and the best-fit constant was $0.106 \pm 0.024$ counts s$^{-1}\left(m_{\text {white }}=22.7_{-0.2}^{+0.3}\right)$. The constant component is most likely due to the host galaxy (Malesani 2010). Figure 4 shows the count rate in the white filter, the XRT count rate, and their ratio.

UVOT used the white filter for the vast majority of the observing time because, for sources with low extinction such as GRB 100418A, it is by far the most sensitive. Low-resolution UVOT spectra using the six filters were obtained for the only two intervals when there were statistically significant detections with multiple filters. All six filters were also used for observations near $T_{0}+1 \mathrm{ks}$, but the exposures were short and only upper limits and marginal detections were obtained. The UVOT spectra were then combined with the contemporaneous XRT spectra to produce broadband SEDs (Figure 5). In addition, 
Table 3

Broadband Spectral Fits

\begin{tabular}{lcccccc}
\hline \hline $\begin{array}{l}t-T_{0} \\
(\mathrm{ks})\end{array}$ & $\begin{array}{c}\text { Exp } \\
(\mathrm{ks})\end{array}$ & $\Gamma$ & $\begin{array}{c}\text { Norm. } \\
\left(10^{-4}\right)\end{array}$ & $\begin{array}{c}n_{\mathrm{H}, \mathrm{z}} \\
\left(10^{20} \mathrm{~cm}^{-2}\right)\end{array}$ & $E_{B-V, z}$ & Filters \\
$(1)$ & $(2)$ & $(3)$ & $(4)$ & $(5)$ & $(6)$ & $(7)$ \\
\hline $5.1-7.3$ & 2.2 & $1.98 \pm 0.09$ & 2.07 & $16 \pm 16$ & $0.056 \pm 0.048$ & All \\
$51.0-92.2$ & 4.4 & $1.95 \pm 0.03$ & 3.01 & 16 & 0.056 & White \\
$573-684$ & 14.8 & $2.15_{-0.08}^{+0.06}$ & 0.19 & 16 & 0.056 & All \\
\hline
\end{tabular}

Notes. (1) Time since the BAT trigger in units of ks; (2) exposure time for the XRT in units of ks; (3) photon spectral index; (4) photons $\mathrm{cm}^{-2} \mathrm{~s}^{-1} \mathrm{keV}^{-1}$ at $1 \mathrm{keV}$. Errors are photon statistical 90\% confidence uncertainties; (5) column density in the host galaxy at $z=0.6235$ in addition to column density in the Milky Way; (6) reddening in the host galaxy in addition to reddening in the Milky Way; (7) UVOT filters included in fit.

results from the UVOT white filter were combined with the XRT spectrum during the interval when the afterglow was the brightest $\left(T_{0}+51 \mathrm{ks}\right.$ to $\left.T_{0}+92 \mathrm{ks}\right)$. The UVOT data points were produced with the tool UVOT2PHA on summed images using the same source and background regions as for the UVOT light curves. The spectral fits were done using the tool XSPEC and the latest UVOT spectral response files (version 104) in CALDB. Table 3 provides a summary of the spectral data.

\subsection{Afterglow Spectrum}

As described above, UVOT and XRT spectra were accumulated for three separate time intervals (Table 3) and then fit with XSPEC. Because of the limited statistical significance of the individual spectral data points, only relatively simple spectral models were used. Each model assumed a power-law continuum with absorption and extinction in both the Milky Way and the host galaxy. The equivalent hydrogen column density in the Milky Way was always set to $4.8 \times 10^{20} \mathrm{~cm}^{-2}$ (Kalberla et al. 2005), and the extinction in the Milky Way was based on a reddening $E_{\mathrm{B}-\mathrm{V}}=0.07 \mathrm{mag}$ (Schlegel et al. 1998). The XSPEC models ZPHABS and ZDUST were used to model absorption and extinction in the host galaxy. A Small Magellanic Cloud-like extinction curve was assumed.

In all cases, a power-law model with a photon index $\Gamma$ of $\sim 2$ provides a good description of the data. The values for the absorption and extinction in the host galaxy were determined only for the spectra accumulated between 5.1 and $7.3 \mathrm{ks}$ after the trigger. This fit provides weak evidence for X-ray absorption in the host galaxy with an $n_{\mathrm{H}}$ of $\sim 1.6 \times 10^{21} \mathrm{~cm}^{-2}$ and extinction corresponding to $E_{\mathrm{B}-\mathrm{V}}=0.056 \mathrm{mag}$. These values were then used for the other two spectra.

\subsection{REM Observations}

REM is a 60-cm diameter fast-reacting telescope with $10^{\circ} \mathrm{s}^{-1}$ pointing speed located at the Cerro La Silla premises of the European Southern Observatory, Chile (Zerbi et al. 2001; Chincarini et al. 2003; Covino et al. 2004a, 2004b). The telescope hosts REMIR, an infrared imaging camera, and ROSS, an optical imager and slitless spectrograph. The two cameras observe simultaneously the same field of view of $10^{\prime} \times 10^{\prime}$ thanks to a dichroic. The telescope observed the field of GRB 100418A from April 19 03:25 UT to 04:34 UT (about $22 \mathrm{ks}$ to $27 \mathrm{ks}$ after the burst), as soon as the field was observable from Chile, as part of an automatic late-time monitoring program. The sequence of observations consisted in the NIR of a sequence of $300 \mathrm{~s}$ exposures in the $z^{\prime}, J, H$, and $K s$ filters, and in the optical a sequence of $60 \mathrm{~s}$ exposures in the $V, R$, and $I$ filters. Data were reduced in a standard way and calibrated by a suitable number of well-exposed Two Micron All Sky Survey objects in the field ${ }^{15}$ for the NIR and Landolt standard stars in the optical. The afterglow was clearly detected in only one frame in the NIR, at 04:14 UT (about $25 \mathrm{ks}$ after the burst) with magnitude $H=14.30 \pm 0.20$. Analysis of the remaining frames did not reveal any source at the afterglow position with $3 \sigma$ upper limit in the best frame at $H>15.2$. There were no UVOT observations at the time of the REM observations, but the expected count rate in the UVOT white filter can be calculated for a specified broadband spectrum. For the best-fit spectrum for $T_{0}$ between 5.1 and $7.3 \mathrm{ks}$ (row 1 in Table 3), a count rate of $12.7 \mathrm{~s}^{-1}$ for $H=14.30$ is expected. For the best-fit spectrum for no extinction in the host galaxy, a count rate of $22.9 \mathrm{~s}^{-1}$ is expected. Both rates are significantly higher than the extrapolation to $6.9 \mathrm{hr}$ of the best fit to white count rates at later times (Figure 3). This and the lack of detections in other frames suggest that the detection is due to a flare.

\section{THEORETICAL INTERPRETATION}

\subsection{Prompt Emission}

GRB 100418A has very low observed prompt energy and luminosity. $E_{\gamma \text {,iso }}$ is $9.9_{-3.4}^{+6.3} \times 10^{50} \mathrm{erg}$, which is lower than any of the 29 GRBs in the sample of Ghirlanda et al. (2004) except for GRB 980425 and two X-ray flashes. It is also near the low end of the distribution for both Swift and pre-Swift bursts compiled by Butler et al. (2010). In a similar fashion, $L_{\gamma}$, iso is $2.1_{-0.6}^{+1.1} \times 10^{50} \mathrm{erg} \mathrm{s}^{-1}$, which is less than any of the $11 \mathrm{GRBs}$ in the sample of Yonetoku et al. (2004) used to establish the $E_{\text {peak }}-L_{\gamma \text {,iso }}$ relationship. This is indicative of the burst being viewed outside the main emission cone, or being intrinsically less energetic.

\subsection{Afterglow}

The X-ray and optical afterglows of GRB 100418A both have a slow rising (plateau) phase and a subsequent power-law decay phase. They very likely originate from the same emission region and within the same spectral regime for $t>T_{0}+5 \mathrm{ks}$ for the following reasons: (1) the ratio of the XRT count rate to UVOT white filter count rate is nearly constant (Figure 4); (2) the UVOT-XRT broadband spectra are consistent with a single power law with the energy index $\beta \sim 1.0$ (Table 3); (3) the UVOT white band and XRT light curve peaks are almost simultaneous. The time $\left(t_{p}\right)$ of the peak count rates in the Swift observations lies between $\sim 50 \mathrm{ks}$ and $\sim 95 \mathrm{ks}$ in both the optical and X-ray bands. During the gap in the Swift observations between $T_{0}+7 \mathrm{ks}$ and $T_{0}+50 \mathrm{ks}$, ground-based observations (Bikmaev et al. 2010) indicate a rapid increase in the optical flux peaking at about $T_{0}+10 \mathrm{ks}$. We attribute this peak to a flare on top of the plateau emission. Such flares are common in the X-ray band and occasionally seen in the optical band (e.g., Greiner et al. 2009).

\subsubsection{Plateau Phase}

Both the X-ray and optical light curves show a long period of time during which the flux is changing slowly. The start and stop times are somewhat uncertain. For the optical band, the plateau begins at least as early as the first observation at $T_{0}+87 \mathrm{~s}$ and ends no later than the peak in the UVOT light curve at

\footnotetext{
15 http://www.ipac.caltech.edu/2mass/
} 
$T_{0}+51 \mathrm{ks}$. With no Swift observations between $T_{0}+7 \mathrm{ks}$ and $T_{0}+51 \mathrm{ks}$, the plateau phase could end anywhere between these two times. In the X-ray band, the transition to the plateau phase is at $T_{0}+600 \mathrm{~s}$, and the plateau appears to end somewhere in the $50-100 \mathrm{ks}$ range. The plateau phase is common in the X-ray band, but the duration in GRB 100418A is toward the high end of the distribution (Evans et al. 2009). The plateau phase in GRB 100418A has some similarities to that of the much brighter GRB 060729 (Grupe et al. 2007). For both bursts, the phase begins at $\sim 500 \mathrm{~s}$ after a steeply falling initial X-ray light curve, and lasts until $\sim 50 \mathrm{ks}$ in both the X-ray and optical bands. In contrast, no optical flare is seen in GRB 060729, and the brightest flux is seen early, rather than late, in the plateau phase of GRB 060729.

A peak between the plateau and late decay phases due to the transition of a characteristic synchrotron frequency in the optical and X-ray band can be excluded, since there is no evidence (Table 3) of spectral evolution around the peak as predicted by the synchrotron forward shock models (for the interstellar medium (ISM) model, see Figure 2 of Sari et al. 1998; for the wind model, see Figure 6 of Zou et al. 2005). Moreover, the difference in the peak times in the optical and X-ray bands is within a factor of $\sim 2$, as stated above. Therefore, the plateau phase and the peak should have a hydrodynamic origin. We also note that the plateau phase cannot be explained in the standard forward shock models through the closure relation. The observed closure relation is $\alpha-1.5 \beta \sim-1.7$, while the predicted relation is $\alpha-1.5 \beta=0 \pm 0.5$ in the standard models (e.g., Zhang et al. 2006).

Energy injection model. One possible explanation for the plateau phase is significant continuous energy injection into the forward shock (Zhang et al. 2006). This can be accomplished by long-lasting operation of the central engine, transferring of the Poynting flux energy to the external shock (Dai \& Lu 1998), or a range in the Lorentz factors of ejecta such that slower ejecta catch up to the decelerated shock (Rees \& Mészáros 1992).

We will start with the first case and assume that luminosity $L(t)=L_{0}\left(t / t_{b}\right)^{-q}$. Table 2 of Zhang et al. (2006) provides the relationship between $\alpha$ and $\beta$ for various GRB models. Since the spectral indices in the X-ray and optical bands are consistent at this time (Section 2.4), we will assume that all the break frequencies for the synchrotron spectrum are below the $\mathrm{X}$-ray band. In this case, $q=2(\alpha+1-\beta) /(1+\beta)=-0.21_{-0.16}^{+0.15}$ for the measured values, $\alpha=-0.23_{-0.14}^{+0.13}$ (Section 2.2) and $\beta=0.98 \pm 0.09$ (Table 3 ). This value for $q$ is marginally consistent with $q=0$ case discussed by Zhang \& Mészáros (2001) for a magnetar-like central engine model. The peak in the light curve corresponds to the cessation of energy injection. The isotropic kinetic energy in the late afterglow is therefore $E_{k, \text { iso }, f}=E_{k, \text { iso }, i}\left(t_{p} / t_{i}\right)^{1-q} \geqslant 10^{53}$ erg, where $t_{p} \sim 50-90 \mathrm{ks}$ is the peak time, the start time of the dominance of the injected energy over the initial energy is $t_{i}<600 \mathrm{~s}$. We assume that the prompt $\gamma$-ray efficiency is $\sim 50 \%$, so that the initial kinetic energy is comparable to the prompt gamma-ray energy release, $E_{k, \text { iso }, i} \sim E_{\gamma, \text { iso }} \sim 10^{51}$ erg.

Models for a range in the Lorentz factors of the ejecta that follow the form of $M(>\gamma) \propto \gamma^{-s}(s>1)$ in which $M$ is the amount of ejected mass moving with Lorentz factors greater than $\gamma$ are equivalent to continuous ejection models for the appropriate value of $s$ (Zhang et al. 2006). For our measured values of $q, s=6.4_{-1.0}^{+1.3}$ for the ISM model. This relatively large $s$ value is disfavored by comparing with the mean value of $\sim 2.5$ and the range of values derived from the previous Swift observations (Table 3 in Nousek et al. 2006). For the wind model, the entire $90 \%$ confidence range in $q$ produces negative values for $s$, which is inconsistent with the model for the range of Lorentz factors.

Off-axis jet model. An alternative explanation for the plateau phase is that the afterglow is being observed from viewing angles slightly outside the jet (e.g., Eichler \& Granot 2006). Depending on the structure of the jet and the off-axis angle, the light curve can have a long initial phase with little change in the flux or a delayed, but a rapidly rising initial phase. If the viewing angle is also outside the $\gamma$-ray jet, then the observed $E_{\gamma \text {,iso }}$ and $E_{\text {peak }}$ will also be reduced. The unusually low values found for GRB 100418A may be consistent with this interpretation.

Let $\theta_{j}$ and $\theta_{v}$ be the half-opening angle of a GRB jet and the viewing angle from the jet axis, respectively. In the following, we adopt the point source approximation for the GRB jet. The isotropic gamma-ray energy release observed within the jet cone is

$$
E_{\gamma, \text { iso }}^{\text {on }}=\frac{\mathfrak{D}(\theta=0)}{\mathfrak{D}\left(\theta=\theta_{v}-\theta_{j}\right)} E_{\gamma, \text { iso }}^{\text {off }} \simeq \gamma_{0}^{2}\left(\theta_{v}-\theta_{j}\right)^{2} E_{\gamma, \text { iso }}^{\text {off }},
$$

where $E_{\gamma \text {,iso }}^{\text {off }}$ is the off-axis energy release at a viewing angle $\theta_{v}>\theta_{j}, \mathfrak{D}(\theta)=\left[\gamma_{0}\left(1-\beta_{0} \cos \theta\right)\right]^{-1}$ is the Doppler factor. In this model, $E_{\gamma \text {,iso }}^{\text {off }}$ is the observed $\gamma$-ray energy release and its value is $\sim 10^{51} \mathrm{erg}$. $\beta_{0}$ and $\gamma_{0}$ are the initial speed (in units of the speed of light) and the Lorentz factor of the jet in the prompt phase. At such early times $\theta_{v}-\theta_{j}>1 / \gamma_{0}$ is easily satisfied, which leads to the second equality of the above equation.

The Lorentz factor evolves as $\gamma=\gamma_{0}\left(t / t_{\mathrm{dec}}\right)^{-3 / 8}$ for $t>t_{\mathrm{dec}}$, if the jet is viewed on-axis and the environment is constant ISM. For forward-reverse external shock models, the deceleration time is $t_{\mathrm{dec}}=\left[\left(3 E_{k \text {,iso }} / 4 \pi \gamma_{0}^{2} n m_{p} c^{2}\right)^{1 / 3} / 2 \gamma_{0}^{2} c\right](1+z)$ in the thin shell case, while $t_{\mathrm{dec}} \simeq T_{90}$ in the thick shell case (Zhang et al. 2003). The transition from the plateau phase to the steep decay phase at $t=t_{p}$ corresponds to the Lorentz factor $\gamma \simeq 1 /\left(\theta_{v}-\theta_{j}\right)$. Therefore, we get $\gamma_{0}\left(\theta_{v}-\theta_{j}\right) \simeq$ $\left(t_{p} / t_{\mathrm{dec}}\right)^{3 / 8}$. For the thick shell case, we can directly estimate $\gamma_{0}\left(\theta_{v}-\theta_{j}\right) \sim 10^{1.5}$, and $E_{\gamma \text {, iso }}^{\text {on }} \sim 10^{54} \mathrm{erg}$. The intrinsic duration of the prompt emission if we observed the jet on-axis is $T_{90}^{\text {on }} \simeq \mathfrak{D}\left(\theta=\theta_{v}-\theta_{j}\right) / \mathfrak{D}(\theta=0) T_{90}^{\text {off }}$ (e.g., Zhang et al. 2009), which is $\sim 10 \mathrm{~ms}$. Such a short intrinsic $T_{90}$ and large intrinsic energy output make the off-axis model less favored compared to the energy injection model, albeit we cannot exclude this possibility. Furthermore, the large value of $\gamma_{0}\left(\theta_{v}-\theta_{j}\right)$ results in a very rapid increase in the plateau phase (Wu et al. 2004), while the observed increase is very slow. This problem may be solved by introducing some structure in the jet profile. For the thin shell case, we can constrain $\gamma_{0}\left(\theta_{v}-\theta_{j}\right) \leqslant 10^{1.5}$, and $E_{\gamma, \text { iso }}^{\text {on }} \leqslant 10^{54} \mathrm{erg}$, because in this case $t_{\mathrm{dec}}>T_{90}$. If the prompt gamma-ray efficiency is $\sim 50 \%$, then the isotropic kinematic energy $E_{k, \text { iso }}$ in the afterglow phase should be of order of $10^{54}$ erg, which is comparable with the late kinematic energy in the energy injection model.

The energy injection model and off-axis jet model cannot be distinguished with complete confidence by the available observational data of GRB 100418A. While both models produce similar energies, we favor the energy injection model because of the difficulties with the off-axis jet model as discussed above.

\subsubsection{Late Decay Phase}

After the peak in the UVOT light curve at about $T_{0}+51 \mathrm{ks}$, both the optical and X-ray light curves generally follow a powerlaw decay model (segment III of the canonical X-ray light 
curve). This decay can be interpreted with the standard afterglow model. The measured values of $\alpha$ and $\beta$ give $\alpha-1.5 \beta=$ $-0.36_{-0.14}^{+0.18}$ in X-ray and $\alpha-1.5 \beta=-0.60_{-0.13}^{+0.16}$ in the optical, which is consistent with the relationship $\alpha-1.5 \beta=-1 / 2$ predicted at frequencies above all the break frequencies in the standard ISM model. The power-law index of shock accelerated electron energy distribution $p$ can be derived from the late optical to X-ray overall spectral index $(\beta=1.15$, Table 3$)$, i.e., $p=2, \beta=2.3$. We can also use the optical and X-ray temporal decay indices to obtain the values of $p$, which are $p=2.16 \pm 0.13$ and $p=2.49_{-0.15}^{+0.17}$, respectively. The above values of $p$ derived in different ways are consistent with each other within $1.5 \sigma$ error bars. We adopt $p=2.3$ in the following calculation.

The typical frequency $v_{m}$, cooling frequency $v_{c}$, and X-ray $\left(v_{X}=10 \mathrm{keV} / h>\max \left\{v_{c}, v_{m}\right\}\right)$ flux density of synchrotron radiation from a GRB jet are (e.g., Granot \& Sari 2002)

$$
\begin{gathered}
v_{m} \simeq 3.5 \times 10^{15} \epsilon_{e}^{2} \epsilon_{B}^{1 / 2} E_{k, \text { iso }, 54}^{1 / 2} t_{5}^{-3 / 2} \mathrm{~Hz}, \\
v_{c} \simeq 4.4 \times 10^{11} \epsilon_{B}^{-3 / 2} n_{0}^{-1} E_{k, \text { iso }, 54}^{-1 / 2}(1+Y)^{-2} t_{5}^{-1 / 2} \mathrm{~Hz}, \\
F_{v_{X}} \simeq 21 \epsilon_{e}^{p-1} \epsilon_{B}^{(p-2) / 4} E_{k, \text { iso }, 54}^{(p+2) / 4}(1+Y)^{-1} t_{5}^{(2-3 p) / 4} \mu \mathrm{Jy},
\end{gathered}
$$

where $\epsilon_{e}$ and $\epsilon_{B}$ are the fractions of the shock energy deposited in electrons and magnetic fields, respectively, and $n$ is the number density of ISM. Since in many previous GRB afterglows, $\epsilon_{e} \gg \epsilon_{B}$ has been derived (e.g., Panaitescu \& Kumar 2002), we approximate the Compton parameter as $Y \simeq\left(\epsilon_{e} / \epsilon_{B}\right)^{1 / 2}$. For model parameters we adopt the notation of $X=X_{n} \times 10^{n}$ in cgs units.

At the beginning of the decay phase, $t \sim 10^{5} \mathrm{~s}$, both $v_{m}$ and $v_{c}$ are below the optical $R$ band $\left(v_{R} \sim 5 \times 10^{15} \mathrm{~Hz}\right)$, which lead to $\epsilon_{e}^{2} \epsilon_{B}^{1 / 2} E_{k, \text { iso }, 54}^{1 / 2} \leqslant 0.1$, and $\epsilon_{e} \epsilon_{B}^{1 / 2} n_{0} E_{k, \text { iso }, 54}^{1 / 2} \geqslant 10^{-3}$, respectively. The observed X-ray flux density at $h v_{X}=10 \mathrm{keV}$ at $t=2 \times 10^{5} \mathrm{~s}$ is $F_{v_{X}} \simeq 10^{-2} \mu \mathrm{Jy}$, which leads to another constraint on model parameters, i.e., $\epsilon_{e}^{p-1.5} \epsilon_{B}^{p / 4} E_{k, \text { iso }, 54}^{(p+2) / 4} \simeq 10^{-3}$. The solution of the above three constraints, assuming $E_{k \text {,iso }} \simeq$ $10^{54} \mathrm{erg}$, is $10^{4 p / 3-10} \leqslant \epsilon_{B} \leqslant 10^{12(p-2.5) /(3-p)} n_{0}^{(4 p-6) /(3-p)}$, or $10^{-7} \leqslant \epsilon_{B} \leqslant 4 \times 10^{-4} n_{0}^{4.6}$. The ISM number density should be $n \geqslant 0.1 \mathrm{~cm}^{-3}$. Therefore, a set of model parameters with $E_{k, \text { iso }} \simeq 10^{54} \mathrm{erg}, n=1 \mathrm{~cm}^{-3}, p=2.3, \epsilon_{e}=0.15$, and $\epsilon_{B}=10^{-4}$ can fit the X-ray and optical light curves in the decay phase, although some parameters cannot be tightly constrained.

Since there is no evidence for a break in the $\mathrm{X}$-ray light curve at late times, any jet break must occur at $t_{b}>2 \times 10^{6} \mathrm{~s}$. This is also supported by the optical observation. The late optical afterglow decayed as $t^{-1.12 \pm 0.10}$, until the host galaxy begins to dominate around $t \sim 10^{6} \mathrm{~s}$. The non-detection of a jet break indicates that the jet's half-opening angle is $\theta_{j}>1 / \gamma \sim$ $14^{\circ} E_{k, \text { iso }, 54}^{-1 / 8} n_{0}^{1 / 8}\left(t / 2 \times 10^{6} \mathrm{~s}\right)^{3 / 8}$, and the collimation-corrected jet's energy is $E_{k, \text { jet }} \simeq\left(\theta_{j}^{2} / 2\right) E_{k \text {,iso }} \geqslant 3 \times 10^{52} E_{k \text {,iso }, 54}^{3 / 4} n_{0}^{1 / 4} \mathrm{erg}$. The jet energy is at the high end of the jet energy distribution of known GRB samples and is comparable to the maximum energy release in magnetar models (Cenko et al. 2010 and references therein). The large jet energy disfavors the off-axis model, because the intrinsic duration of $\sim 10 \mathrm{~ms}$ in this model would classify GRB 100418A as a short energetic GRB, which is rare event.

\section{SUMMARY}

GRB 100418A is a long burst $\left(T_{90} \sim 8 \mathrm{~s}\right)$ with unusual, but similar, light curves in the X-ray and optical bands. A long plateau phase begins at $T_{0}+600 \mathrm{~s}$ in the X-ray band and lasts until later than $T_{0}+7 \mathrm{ks}$ and perhaps until $T_{0}+51 \mathrm{ks}$ or even longer. The optical flux appears to be flat or rising from the earliest observation starting at $T_{0}+87 \mathrm{~s}$ until at least $T_{0}+7 \mathrm{ks}$, and the peak UVOT flux was seen at $T_{0}+51 \mathrm{ks}$. The afterglow then transitions to a late power-law decay phase, which lasts until at least $T_{0}+1.0 \mathrm{Ms}$ in the optical and $T_{0}+2.0 \mathrm{Ms}$ in the X-ray band with no evidence for a jet break. Joint spectral fits to the XRT and UVOT data are consistent with a power-law model with an index $\beta \sim 1$. Long plateau phases with optical light curves peaking at tens of ks have been seen in only a handful of other GRBs, and they have been interpreted as resulting from continued energy injection (e.g., GRB 060729, Grupe et al. 2007) or off-axis viewing of the jet (e.g., GRB 081028, Margutti et al. 2010). The long plateau phase of GRB 100418A is most likely due to continued Poynting flux energy injection with roughly constant luminosity. The total isotropic kinetic energy at the end of the plateau phase is more than $\sim 10^{53} \mathrm{erg}, 100$ times the initial value. The lack of a jet break indicates a jet's half-opening angle of at least $\sim 14^{\circ}$, and a relatively high jet energy after collimation correction, $E_{\text {jet }} \geqslant 10^{52} \mathrm{erg}$. This jet energy is near the high end of the distribution for known GRBs.

This work made use of data supplied by the UK Swift Science Data Centre at the University of Leicester and the High Energy Astrophysics Science Archive Research Center, provided by NASA's Goddard Space Flight Center. B.Z., X.F.W., and E.W.L. acknowledge NASA NNX09AT66G, NNX10AD48G, and NSF AST-0908362 for support. X.F.W. and E.W.L. are also supported by National Basic Research Program of China (973 Program 2009CB824800). P.A.E., J.P.O., and C.P. acknowledge STFC support.

\section{REFERENCES}

Antonelli, S., et al. 2010, GCN Circ., 10620

Band, D., et al. 1993, ApJ, 413, 281

Barthelmy, S. D., et al. 2005, Space Sci. Rev., 120, 143

Bikmaev, I., et al. 2010, GCN Circ., 10635

Breeveld, A. A., et al. 2010, MNRAS, 406, 1687

Burrows, D. N., et al. 2005, Space Sci. Rev., 120, 165

Butler, N. R., \& Kocevski, D. 2007, ApJ, 663, 407

Butler, N. R., et al. 2010, ApJ, 711, 495

Cash, W. 1979, ApJ, 228, 939

Cenko, S. B., et al. 2010, ApJ, 711, 641

Chincarini, G., et al. 2003, Messenger, 113, 40

Covino, S., et al. 2004a, Astron. Nachr., 325, 543

Covino, S., et al. 2004b, Proc. SPIE, 5492, 1613

Covino, S., Fugazza, D., Antonelli, L. A., \& Malesani, D. 2010, GCN Circ., 10646

Cucchiara, A., \& Fox, D. B. 2010, GCN Circ., 10624

Dai, Z. G., \& Lu, T. 1998, A\&A, 333, L87

Della Valle, M., et al. 2006, Nature, 444, 1050

Eichler, D., \& Granot, J. 2006, ApJ, 641, L5

Evans, P. A., et al. 2007, A\&A, 469, 379

Evans, P. A., et al. 2009, MNRAS, 397, 1177

Evans, P. A., et al. 2010, A\&A, 519, 102

Gehrels, G., et al. 2004, ApJ, 611, 1005

Ghirlanda, N., Ghisellini, G., Lazzati, D., \& Firmani, C. 2004, ApJ, 616, 331

Goad, M. R., et al. 2007, A\&A, 476, 1401

Granot, J., \& Sari, R. 2002, ApJ, 568, 820

Greiner, J., et al. 2009, ApJ, 693, 1912

Grupe, D., et al. 2007, ApJ, 662, 443

Guidorzi, C., et al. 2007, A\&A, 474, 793 
Kalberla, P. M. W., Burton, W. B., Hartmann, D., Arnal, E. M., Bajaja, E., Morras, R., \& Pöppel, W. G. L 2005, A\&A, 440, 775

Kaneko, Y., Preece, R. D., Briggs, M. S., Paciesas, W. S., Meegan, C. A., \& Band, D. L. 2006, ApJS, 166, 298

Kouveliotou, C., Meegan, C. A., Fishman, G. J., Bhat, N. P., Briggs, M S., Koshut, T. M., Paciesas, W. S., \& Pendleton, G. N. 1993, ApJ, 413, L101

Malesani, D. 2010, GCN Circ., 10621

Mangano, V., et al. 2007, A\&A, 470, 105

Margutti, R., et al. 2010, MNRAS, 402, 46

Marshall, F. E., et al. 2010, GCN Circ., 10612

Mészáros, P. 2006, Rep. Prog. Phys., 69, 2259

Nousek, J. A., et al. 2006, ApJ, 642, 389

Oates, S. R., et al. 2009, MNRAS, 395, 490

Osborne, J. P., Beardmore, A. P., Evans, P. A., \& Goad, M. R. 2010, GCN Circ. 10614

Pagani, C., Beardmore, A. P., \& Marshall, F. E. 2010, GCN Circ. 10622

Panaitescu, A., \& Kumar, P. 2002, ApJ, 571, 779

Panaitescu, A., \& Vestrand, W. T. 2008, MNRAS, 387, 497

Piran, T. 2005, Rev. Mod. Phys., 76, 1143

Poole, T. S., et al. 2008, MNRAS, 383, 627

Rees, M. J., \& Mészáros, P. 1992, MNRAS, 258, 41p

Roming, P. W. A., et al. 2005, Space Sci. Rev., 120, 95
Sakamoto, T., et al. 2008, ApJ, 679, 570

Sakamoto, T., et al. 2009, ApJ, 693, 922

Sari, R., Piran, T., \& Narayan, R. 1998, ApJ, 497, L17

Schlegel, D. J., Finkbeiner, D. P., \& Davis, M. 1998, ApJS, 500, 525

Shen, R., Kumar, P., \& Piran, T. 2010, MNRAS, 403, 229

Siegel, M. H., \& Marshall, F. E. 2010, GCN Circ., 10625

Stanek, K. Z., et al. 2007, ApJ, 654, L21

Troja, E., et al. 2007, ApJ, 665, 599

Ukwatta, T. N., et al. 2010, GCN Circ., 10615

Woosley, S. E. 1993, ApJ, 405, 273

Woźniak, P. R., Vestrand, W. T., Wren, J. A., White, R. R., Evans, S. M., \& Casperson, D. 2006, ApJ, 642, L99

Wu, X. F., Dai, Z.-G., Huang, Y.-F., \& Ma, H.-T. 2004, Chin. J. Astron. Astrophys., 4, 455

Yonetoku, D., Murakami, T., Nakamura, T., Yamazaki, R., Inoue, A. K., \& Ioka, K. 2004, ApJ, 609, 935

Zerbi, F. M., et al. 2001, Astron. Nachr., 322, 275

Zhang, B., Fan, Y. Z., Dyks, J., Kobayashi, S., Mészáros, P., Burrows, D. N., Nousek, J. A., \& Gehrels, N. 2006, ApJ, 642, 354

Zhang, B., Kobayashi, S., \& Mészáros, P. 2003, ApJ, 595, 950

Zhang, B., \& Mészáros, P. 2001, ApJ, 552, L35

Zhang, B., et al. 2009, ApJ, 703, 1696

Zou, Y. C., Wu, X. F., \& Dai, Z. G. 2005, MNRAS, 363, 93 